\title{
Software for Reaction-Time Measurement and its Application for the Evaluation of Patient's Recovery after the Stroke
}

\author{
Mirjana Dejanović \\ Department of Physiology \\ Faculty of Medicine, University of Priština \\ Kosovska Mitrovica, Serbia \\ mirjana.dejanovic@gmail.com \\ Igor Dejanović \\ Department of Computing and Control Engineering \\ Faculty of Technical Sciences, University of Novi Sad \\ Novi Sad, Serbia \\ igord@uns.ac.rs
}

\begin{abstract}
The purpose of this study was to present a simple, cross-platform and easy to use software for reaction-time task and to find out the possibility of its application to the evaluation of patient's recovery after the ischemic stroke.
\end{abstract}

Keywords—reaction-time; software; stroke;

\section{INTRODUCTION}

The use of neuropsychological tests in patients after the stroke has become an obligatory part of the diagnostic protocol. Considering that the acute phase of a stroke greatly hinders the implementation of a comprehensive neuropsychological battery in most of these patients, emphasizes the importance of rapid tests for the assessment of cognitive status. Application of the method of reaction time is a quick and easy way to enable monitoring of mental processes that underlie a perception, attention, memory and action. The reaction time is the process that involves the receipt of information, it's processing, decision-making and response-execution of motor acts [1]. The time interval from the moment of simple or complex stimuli presentation to the moment of the motor response reflects the speed of neurophysiological, cognitive and information processes that occurs as a response to the application of stimuli to the respondent sensory system.

Simple reaction time (SRT) includes reaction to the known stimulus and the same response is expected in subsequent attempts, thus the subject is able to preprogram the response move [2]. The response in the choice reaction time (CRT) is unknown until the appearance of the imperative stimulus, when the subject can plan, i.e. preprogram and initiate a response. In other words, the motor response was determined with at least 2 parameters where one of them can be changed between trials. In the choice reaction time, a response is not known in advance and, therefore, advantages related to the anticipation cannot be used. Choice reaction time includes an analysis of stimuli and selection response, i.e. central cognitive processing [3].

A reaction time method test reflects the level of readiness of cognitive neural mechanisms, thus their use in the study of reaction speed in a variety of situations is tenable, both in healthy subjects and various diseases. Determining the value of simple and choice reaction time gives an insight into the functional state of the general reactivity of the individual in given time and circumstances.

Although a need for easily accessible good quality software for different types of reaction time is apparent we have found that the existing software is either proprietary and expensive or hard to use. Therefore, we have implemented easy to use crossplatform software for reaction time which will be presented in this paper. The software is in use at the Department for neurological disease at the Rehabilitation centre Novi Sad in Serbia and the Faculty of Medicine in Kosovska Mitrovica.

The aim of this work is to present our software for reaction time and its possibility for assessment of cognitive recovery of patients after the stroke.

\section{RELATED WORK}

There is not much reaction-time software that is freely available, easy to use and cross-platform. 


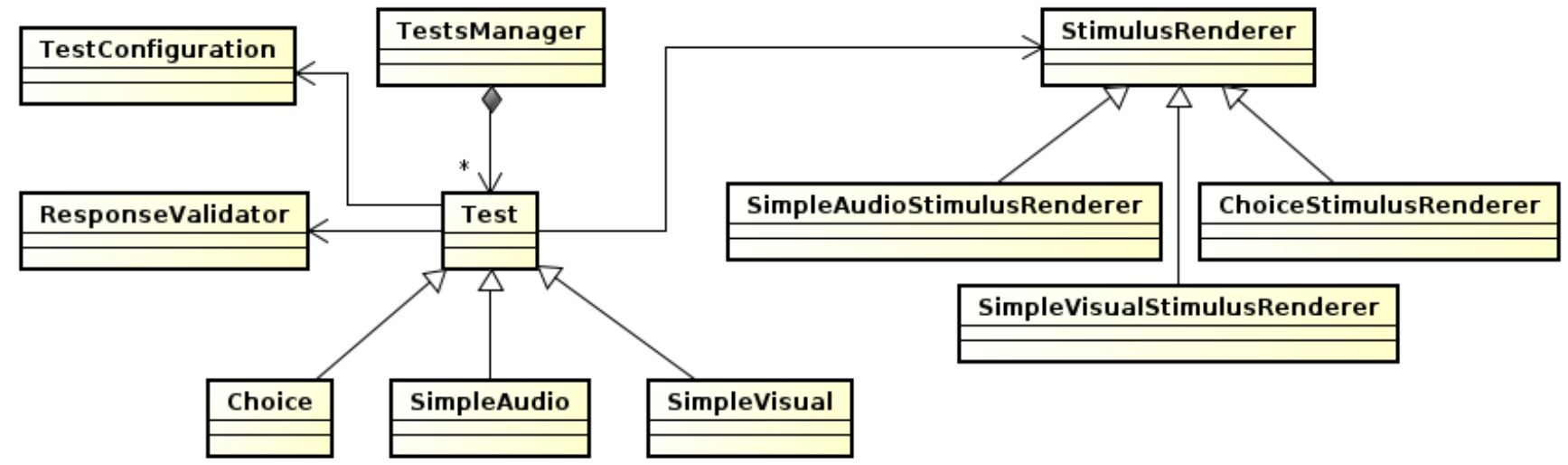

Fig. 1. Test and stimulus renderer class hierarchy

Commercially available software that can be used for reaction-time tasks, beside their price which might be a limiting factor to their use, are usually constrained to particular platform which hinders its use in the heterogeneous environments. DirectRT [4] is commercial reaction time software developed by Empirisoft. It claims to be very precise and easy to use. It is available on Windows platform only. Inquisit Lab [5] is also commercial software that can be configured to perform simple and choice reaction time tests.

There are freely available libraries and tools that can be used to help in building of reaction-time tests, but usually require at least some basic level of programming skills. PsychoPy [6] is a library and a toolset for Python programming language that can be used to build sophisticated psychological test, but require an understanding of Python language and PsychoPy application programming interface. OpenSesame [7] is another option where an experiment can be described using graphical user interface thus enabling less experienced users to built their experiments.

Tests of reaction time are proposed in the diagnosis of cerebral damage as indicators of its severity and extent [8]. In patients with stroke, simple and choice reaction time will be used for the assessment of cognitive status in the acute phase of stroke and also during the recovery [9].

Some studies show that simple and choice reaction times have a predictive role because faster choice reaction time in the acute phase of the stroke was significantly associated with better quality of life at 12 months [10]. The same authors found that simple and brief computerized assessment of attentional function in acute stroke is possible and is related to longer term attentional and cognitive performance. In $[9,11]$ authors have studied the efficacy of a computer-assisted reaction training on various attentional and cognitive functions in stroke patients with lateralized cortical lesions. All patients showed significant training effects for a number of attention functions, but not for vigilance, and there was no generalization of the training effects to more general cognitive functions [11]. However, training effects were more pronounced in the patients with lesions in the left hemisphere (or left brain-damaged group).

\section{MATERIALS AND METHODS}

The study included 60 patients (31 men and 29 women, mean age $59.7 \pm 8.8$ years, range $50-70$ years) with acute brain infarction. Patients were recruited from the Department for neurological disease at the Rehabilitation centre Novi Sad Serbia. The control group consisted of 30, age and sex matched healthy volunteers (14 men and 16 women, mean age 58.5 \pm 8.2 years, range 50-72 years. The protocol of the study was approved by the local Ethics Committee, in accordance with the principles of the Declaration of Helsinki. An informed consent was obtained from all patients, before they were enrolled into the study.

Simple and choice reaction time test was performed using a specially constructed computer program.

Simple reaction time test consisted of two blocks of tasks. The first being a visual stimuli task while the second was the audible. For simple reaction time task subjects were instructed to press Spacebar as fast as they can when the white circle was presented on the screen (visual stimuli), or the tone was played (audible stimuli). For each block stimuli were applied in a series of 30 repetitions where the time interval between two stimuli presentation varied between 1.5 and 3 seconds. For each subject a visual stimuli test was performed before the audible. From the 30 RT values for each serie (visual and audible) and average value vas calculated and registered for the subject.

Choice reaction time test consisted of three blocks of tasks with 2, 4 or 6 choices each. Choices were given as digits 1-6 on the screen and subjects were instructed to press corresponding key on the keyboard when the digit is highlighted. As with simple reaction task, for each block, stimuli were applied in a series of 30 repetitions where the time interval between two stimuli presentations varied between 1.5 and 3 seconds.

\section{REACTION TIME SOFTWARE}

Reaction is implemented in Java programming language and thus can run on every platform for which a Java runtime exists. This spans a wide variety of operating system environments. 
Reaction currently supports two simple tests (visual and audible) and a choice reaction time test. From the graphical interface, an operator chooses a test to run. Tests are run in full-screen mode. Test session consists of one or more trial series. Each series consists of configured number of trials. At the beginning of each series, a user is presented with the short introduction where she is explained what she is supposed to do. Optionally, before each real series a practice series can be run. Practice series performs the same as the real one but the reaction data will not be collected and the number of trials will be smaller. In each trial, a subject must respond to the stimuli usually by pressing specific button on the keyboard. If the subject presses the button before the stimulus has appeared or presses the wrong button an error tone will be played and that particular trial will be marked as error. The number of erroneous trials is also an interesting datum to observe. At the end of each series collected data are displayed on the screen. At the end of the test session, an Excel file is written to the disk containing all collected reaction time data.

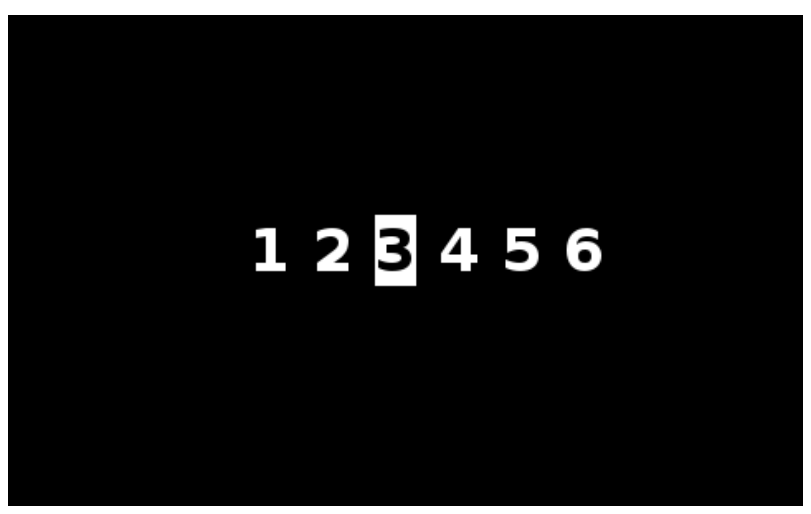

Fig. 2. Choice reaction time for 6 different stimuli

The main concept of Reaction is a test represented as Test class in Fig. 1. The tests instances are contained inside TestManager. Currently, tests are specified in Java by inheriting Test class but following versions will provide the means to specify tests using external textual description based on specially constructed language (i.e. Domain-Specific Language [12]). There are three types of test at the moment: SimpleAudio, SimpleVisual, and Choice.

Stimuli presentation is done by the instances of classes inheriting StimulusRenderer class. The job of stimuli renderers is to present stimulus to the user. The reaction is validated by the ReactionValidator instance.

SimpleAudio test type will play a tone of a specific frequency at random interval from the given minimum and maximum interval in milliseconds. Reaction validator will expect a press on spacebar to happen after the tone starts playing. The time that passes from the beginning of the tone to the key press will be recorded as RT.

SimpleVisual test type is similar to the SimpleAudio but will present a white circle at the center of the screen. Before a circle is presented a white + sign (i.e. fixation point) will be presented to the user. This is needed for the user to better focus her attention while expecting a stimulus to appear.

Choice class implements a choice reaction time which displays a configurable number of digits from left to right centered horizontally and vertically on the screen. The stimulus, in this case, will be a random highlight of one of the digit as shown in Fig. 2. Expected response is pressing a corresponding digit on the keyboard. Choice can be configured with 2-10 digits to appear, but it is usually configured for 2, 4, 6 digits. Recorded reaction time will be a time that passes from the highlight to the press on the right digit on the keyboard. If the user presses the wrong key the trial will be recorded as an error.

TABLE I. RESULTS OF CHOICE REACTION TIME TEST FOR PATIENTS AND CONTROL GROUP AT ACUTE PHASE OF THE STROKE

\begin{tabular}{|c|c|c|c|c|}
\hline CRT & Patients [ms] & Controls [ms] & t & p \\
\hline 2 & 710.2 & 486.4 & -4.33 & $<0.01$ \\
\hline 4 & 985 & 705 & -4.23 & $<0.01$ \\
\hline 6 & 1305 & 870 & -5.25 & $<0.01$ \\
\hline
\end{tabular}

The results obtained using choice reaction time test, presented in Table I, shows that the RT values for patients with ischemic stroke at the beginning of the study greater than the values of the subjects in the control group and that difference is statistically significant. At second registration, three months after the first registration a statistically significant recovery of RT values have been observed.

\section{CONCLUSION}

We have described cross-platform software for performing simple and choice reaction-time tasks which we used to evaluate patient's recovery after the ischemic stroke. We have found that using a computer-based reaction time test is a quick and easy way to assess the cognitive abilities of patients after an ischemic stroke. This may be a significant aid in the forecast of their recovery.

\section{REFERENCES}

[1] Pachela R.G., "The interpretation of reaction time in informationprocessing research", In Human information procesing - tutorials in performance and cognition.Ed. Barry H. Kantowitz. New York, 1978.

[2] Kohfeld D.L., "Simple reaction as a function of stimulus intensity in decibels of light and sound", Journal of Experimental Psychology, 1971;88:251-257.

[3] Tenenbaum G., Yuval R., Elbaz G., Bar-Eli M. and Weinberg R., "The relationship between cognitive characteristics and decision making", Can J Appl Physiol, 1993, Mar; 18 (1):48-62.

[4] "Direct RT", Empirisoft, http://www.empirisoft.com/directrt.aspx, online, accessed January, 24. 2015.

[5] "Inqusit Lab", Milisecond, http://www.millisecond.com/, online, accessed January, 24. 2015.

[6] Peirce, JW, "PsychoPy - Psychophysics software in Python", J. Neurosci Methods, 162(1-2):8-13. 2007.

[7] Mathôt, S., Schreij, D., and Theeuwes, J. "OpenSesame: An opensource, graphical experiment builder for the social sciences". Behavior 
ICIT 2015 The $7^{\text {th }}$ International Conference on Information Technology

doi:10.15849/icit.2015.0027 C ICIT 2015 (http://icit.zuj.edu.jo/ICIT15)

Research Methods, 44(2), 314-324. doi:10.3758/s13428-011-0168-7. 2012

[8] Dee H. L. and Van Allen M. W., "Psychomotor testing as an aid in the recognition of cerebral lesions". Neurolog.v 22, 845-848, 1982.

[9] Cumming TB1, Brodtmann A, Darby D. and Bernhardt J.,"Cutting a long story short: reaction times in acute stroke are associated with longer term cognitive outcomes". J Neurol Sci. 2012 Nov 15;322(1-2):102-6.
[10] Cumming TB, Brodtmann A, Darby D, Bernhardt J., "The importance of cognition to quality of life after stroke", J Psychosom Res. 2014 Nov;77(5):374-9.

[11] Walter Sturm and Klaus Willmes, "Efficacy of a reaction training on various attentional and cognitive functions in stroke patients", Neuropsychological Rehabilitation: An International Journal Volume 1, Issue 4, 1991 pages 259-280

[12] Fowler, M., "Domain-Specific Languages", Addison-Wesley Professional, 